She received a second dose of IVIG and 5 pulses of methylprednisolone but developed progressive neurological compromise, catatonia, and difficulty swallowing, requiring nasojejunal tube feedings.

Plasmapheresis was initiated with a regular response. She required electroconvulsive therapy (ECT) for catatonia management. After the first ECT, she developed focal left frontal status epilepticus, was switched to phenytoin and valproic acid with good response. ECT was restarted after no crisis where detected, completing 8 sessions. Rituximab was initiated, completing four doses. There was a slow but steady recovery in her mental and neurological status, no signs of catatonia, persisted with an altered short memory, language difficulties, slightly inattentive, with emotional lability, recovered a normal gait and oral feedings. Paraneoplastic markers where all negative. She was discharged after 53 days of hospitalization with prednisone, valproic acid, Quetiapine, and lorazepam.

This patient presents a severe case of Autoimmune Encephalitis, with severe psychiatric symptoms and other autoimmune manifestations. Usually, there is a good response after treatment with IVIG and/or methylprednisolone. Rituximab was chosen to reduce de antibody production. Follow up as an outpatient, she has had no relapses and continues to recover.

\section{GP51 PANDORAEA SPUTORUM IN A PAEDIATRIC CYSTIC FIBROSIS PATIENT}

${ }^{1}$ Diana Bordalo*, ${ }^{2}$ Mariana Branco, ${ }^{3}$ Rudi Carvalho, ${ }^{4}$ Luísa Vaz. ${ }^{1}$ Centro Hospitalar do Médio Ave, Vila Nova de Famalicão, Portugal; ${ }^{2}$ Unidade Local de Saúde do Alto Minho, Viana do Castelo, Portugal; ${ }^{3}$ Centro Hospitalar do Algarve, Faro, Portugal; ${ }^{4}$ Hospital Pediátrico Integrado do Centro Hospitalar de São João, Porto, Portugal

\subsection{6/archdischild-2019-epa.117}

Introduction Cystic fibrosis (CF) is the most frequent fatal genetic disease among caucasians. It is caused by mutations in the cystic fibrosis transmembrane conductance regulator (CFTR) protein, which is found in all exocrine tissues, leading to thick, viscous secretions in several organs. The major cause of morbidity and mortality in CF's patients is lung function's deterioration due to pathogenic colonization with several bacteria. One emerging multidrug resistant pathogen that has been identified in CF is Pandoraea sputorum, a non-fermenting Gram negative bacteria.

Case report A 8-year-old boy, diagnosed with CF at 4 years old (homozygous genotype F508del; sweat test positive: 150 $\mathrm{mEq} / \mathrm{L}$ ) was admited to the hospital for intravenous treatment of Pandoraea sputorum bronchopulmonary colonization. This pathogen was identified by matrix-assisted laser desorption ionization-time of flight mass spectrometry. He had a history of Pseudomonas aeruginosa lung colonization at 4 years old, and of Stenotrophmonas maltophila at 7 years old, all of which had been eradicated after intravenous antibiotic treatment. All prior respiratory exacerbations were also treated empirically. His previous pulmonary function test revealed a forced expiratory volume in 1second (FEV1) of $89 \%$. He was assymptomatic before, and throughout his inpatient stay, that lasted for 14 days while he received intravenous imipenem $(80 \mathrm{mg} / \mathrm{Kg}$ per day). Two weeks after being discharged he was assessed at a paediatric pneumology appointment, remaining without symptoms, and with a FEV1 of $88 \%$. His follow-up sputum culture was negative for Pandoraea sputorum.

Discussion In this clinical case the authors followed the protocol for early eradication, treating when the pathogen is first acquired, regardless of age or clinical signs or symptoms. This was based on several case reports that compared Pandoraea sputorum to Pseudomonas aeruginosa in terms of pathogenicity and decline of lung function. There is still a need to better understand this microorganism, and its correlation with disease outcomes, that can arise from the description of more case reports.

\section{GP52 THE UNSUSPECTED HUMAN METAPNEUMOVIRUS RESPONSIBLE FOR A SEVERE ERYTHEMA MULTIFORME: AN UNUSUAL ASSOCIATION}

${ }^{1}$ Rosa Canestrale, 'Rossella Giorgio, 'Sofia Siena, ${ }^{2}$ Carmela De Meco, 2 Irene Rutigliano, ${ }^{1}$ Agostino Petraccaro, ${ }^{1}$ Enrica Manca*, ${ }^{1}$ Angelica Dirodi, ${ }^{2}$ Michele Carmine Sacco, ${ }^{2}$ Massimo Pettoello Mantovani. 'Residency program in Pediatrics, University of Foggia, Foggia, Italy; ${ }^{2}$ Department of Pediatrics, Pediatric Unit, 'Casa Sollievo della Sofferenza' Scientific Institute, University of Foggia, San Giovanni Rotondo, Italy

10.1136/archdischild-2019-epa.118

Erythema Multiforme (EM) is an acute immune-mediated condition characterized by the appearance of typical target-like lesions on the skin. They most commonly appear in a symmetrical distribution on the extensor surfaces of the acral extremities and subsequently spread in a centripetal way. EM 'major' involves oral, genital and ocular mucosae with erosions or bullae. Although cutaneous lesions are usually asymptomatic, EM can be caused by drugs, autoimmune disease, malignancy, irradiation, sarcoidosis and in 90 percent of cases by infections (viral, bacterial, fungal). Herpes Simplex virus is the most frequent etiologic agent. Mycoplasma Pneumoniae infection is another important cause of EM, particularly in children. Laboratory findings are not specific and clinical finding are necessary for diagnosis. A skin biopsy should be performed when the diagnosis is unclear. CASE REPORT: 14 year old male came to our attention for the appearance of cutaneous lesions, accompanied by high fever. The skin appeared almost entirely affected by roundish, sharp, erythematous lesions, some of these with evident 'coccard' sign, other ecchymotic with hemorrhagic nuance, confluent to the trunk in large patches. No recent history of infections or drugs. Laboratory findings showed a neutrophilia (N 8810/mcl) and eosinophilia (E 980/mcl) and high inflammatory indices (PCR $4.75 \mathrm{mg} / \mathrm{dl}$, ferritin $517 \mathrm{~kg} / \mathrm{ml}$ ). Peripheral smear, autoimmunity, virological and bacterial screening and instrumental examinations were negative. On the third day of admission, he performed a nasal swab (Multiplex) due to the appearance of rhinorrhea and cough. It was positive for Human Metapenumovirus (HM). On the seventh day, there was a new poussé of erythematous, itchy, coccard element on the whole body surface. He was treated with antihistaminic, steroid and antibiotic therapy with gradual rash regression, desquamation of skin lesions and defervescence. In literature it is known that $\mathrm{HM}$ is a common cause of upper respiratory tract infection in children. However, no further cases are reported regarding the possible relationship between skin lesions and HM. In our case the only laboratory finding associate to the EM was a positive RT-PCR for HM. This observation could lead to further scientific evaluations. 